\title{
COBERTURA DE ASEGURAMIENTO EN SALUD: EL CASO PERUANO DESDE LA LEY DE ASEGURAMIENTO UNIVERSAL
}

\author{
Edward Mezones-Holguín ${ }^{1,2, a}$, Elard Amaya ${ }^{1, b}$, Luciana Bellido-Boza ${ }^{3,4, c}$, Benoit Mougenot ${ }^{1, \mathrm{~d}}$, \\ Juan P. Murillo ${ }^{3,5, e}$, José Villegas-Ortega ${ }^{3,6, f}$, José Carlos Del Carmen Sara ${ }^{5,7,9}$
}

\begin{abstract}
RESUMEN
Objetivo. Describir la evolución de la cobertura de aseguramiento en salud (CAS) en Perú para el periodo 2009-2017 y evaluar los principales factores demográficos, sociales y económicos asociados. Materiales y métodos. Realizamos un análisis secundario de la Encuesta Nacional de Hogares. Para cada año estimamos la CAS global, del Seguro Integral de Salud (SIS) y del Seguro Social en Salud (EsSalud), y realizamos pruebas de tendencias anuales. Para los años 2009 (Ley de Aseguramiento Universal en Salud), 2013 (reforma del sector salud) y 2017, construimos una variable politómica del tipo de aseguramiento (SIS/EsSalud/No asegurado) y estimamos razones relativas de prevalencia (RRP) con intervalos de confianza (IC) al 95\% mediante modelos logísticos multinomiales para muestras complejas. Resultados. Observamos un incremento en la CAS global (2009: 60,5\%; 2013: 65,5\%; 2017: 76,4\%), en el SIS (2009: 34\%; 2013: 35,4\%; 2017: 47\%) y en EsSalud (2009: 22,8\%; 2013: 26,4\%; 2017: 26,3\%). Observamos que ser mujer aumentó la posibilidad de afiliación al SIS (RRP=2009: 1,64 y 2017: 1,53), mientras que tener entre 18 y 39 años, residir Lima Metropolitana y ser no pobre redujeron esa posibilidad $(R R P=2009$ : 0,16 y $2017: 0,31 ; 2009: 0,17$ y $2017: 0,37 ; 2009: 0,51$ y $2017: 0,53$; respectivamente). Por su parte, ser mujer, tener más de 65 años, ser del ámbito urbano, residir en Lima Metropolitana y ser no pobre aumentó la probabilidad de estar afiliados a EsSalud (RRP=2013: 1,12 y 2017: 1,24; $2013: 1,32$ y 2017 : 1,34; 2009: 2,18 y 2017: 2,08; 2009: 2,14 y 2017: 2,54; 2009: 3,57 y 2017: 2,53; respectivamente). Conclusiones. La CAS ha incrementado durante el periodo 2009-2017. No obstante, las características de la población asegurada difieren de acuerdo con el tipo de seguro.
\end{abstract}

Palabras clave: Cobertura Universal en Salud; Seguridad Social; Protección contra Riesgos Financieros; Política Pública; Perú. (Fuente: DeCS BIREME).

\section{HEALTH INSURANCE COVERAGE: THE PERUVIAN CASE SINCE THE UNIVERSAL INSURANCE ACT}

\begin{abstract}
Objective. To describe the trends in health insurance coverage (HIC) in Peru during the period 2009-2017 and evaluate associations with demographic, social and economic factors. Materials and Methods. We carried out a secondary data-analysis from the Peruvian National Household Survey. For each year, we estimated the global HIC, for the Integral Health Insurance (SIS) and the Social Security system (EsSalud). In addition, we performed a trend analysis. For 2009 (Universal Health Insurance Act), 2013 (health care reform act) and 2017, we used a polytomous variable for the insurance type (SIS/EsSalud/Non-affiliated). We performed logistic multinomial regressions to estimate relative prevalence ratios (RPR) and their $95 \% \mathrm{Cl}$ with correction for complex sampling. Results. We observed an increasing trend in the global HIC (2009:60.5\%; 2013:65.5\%; 2017:76.4\%), SIS coverage (2009:34\%; 2013:35.4\%; 2017:47\%) and EsSalud coverage (2009:22.8\%; 2013:26.4\%; 2017:26.3\%). Multinomial logistic regressions showed that being a woman increased the likelihood to be affiliated to the SIS (RPR= 2009:1.64 and 2017:1.53), while people between 18 and 39 years old, living in Lima Metropolitan area under non-poverty conditions reduced the likelihood to be affiliated to the SIS (RPR= 2009:0.16 and 2017:0.31; 2009:0.17 and 2017:0.37; 2009:0.51 and 2017:0.53; respectively). Furthermore, being a woman, 65 years old or over, living in urban Lima, and under non-poverty conditions increased the likelihood of being affiliated with the EsSalud (RPR= 2013:1.12 and 2017:1.24; 2013:1.32 and 2017:1.34; $2009: 2.18$ and 2017:2.08; 2009:2.14 and 2017:2.54; 2009:3.57 and 2017:2.53; respectively). Conclusions. HIC has increased during the period 2009-2017. However, the characteristics of those affiliated are different between the various types of health insurance.
\end{abstract}

Keywords: Universal Health Coverage; Financial Risk Protection; Social Security; Public Policy; Peru. (Source: MeSH NLM).

\footnotetext{
Universidad San Ignacio de Loyola, Vicerrectorado de Investigación, Centro de Excelencia en Investigaciones Económicas y Sociales en Salud. Lima, Perú. Epi-gnosis Solutions. Piura, Perú.

Superintendencia Nacional de Salud, Intendencia de Investigación y Desarrollo. Lima, Perú.

Universidad Peruana de Ciencias Aplicadas, Facultad de Ciencias de la Salud. Lima, Perú.

Universidad Nacional Mayor de San Marcos, Facultad de Medicina de San Fernando. Lima, Perú.

Universidad Peruana de Ciencias Aplicadas, Escuela de Postgrado. Lima, Perú.

Superintendencia Nacional de Salud, Alta Dirección. Lima, Perú.

Médico cirujano, maestro en ciencias en Epidemiología Clínica; ${ }^{\mathrm{b}}$ economista; ${ }^{\mathrm{c}}$ licenciada en Nutrición; ${ }^{\mathrm{d}}$ economista, doctor en Economía; ${ }^{\mathrm{e}}$ médico cirujano;

${ }^{\mathrm{f}}$ licenciado en Computación, magíster en Ingeniería de Sistemas; $\mathrm{g}$ médico cirujano, especialista en Neurología, doctor en Salud Pública.

Recibido: 22/10/2018 Aprobado:20/03/2019 En línea: 28/06/2019
}

Citar como: Mezones-Holguín E, Amaya E, Bellido-Boza L, Mougenot B, Murillo JP, Villegas-Ortega J, Del Carmen Sara JC. Cobertura de aseguramiento en salud: el caso peruano desde la Ley de Aseguramiento Universal. Rev Peru Med Exp Salud Publica. 2019;36(2):196-206. doi: http://dx.doi.org/10.17843/rpmesp.2019.362.3998. 


\section{INTRODUCCIÓN}

En el marco de la cobertura universal en salud (CUS), los derechos en Salud (DES) son fundamentales, aunque existen barreras que pueden limitar su ejercicio ${ }^{(1)}$. En el contexto de los objetivos de desarrollo sostenible, la CUS constituye una expresión práctica de los DES ${ }^{(2-3)}$. Se han dado iniciativas, principalmente gubernamentales, que apuntan al logro de la CUS, donde el Aseguramiento Universal en Salud (AUS) cobra cardinal importancia como un elemento de protección financiera al reducir especialmente el impacto en el gasto catastrófico en salud ${ }^{(4-6)}$. Consecuentemente, el AUS contribuye a la consolidación de la CUS.

El avance del AUS es relevante para las sociedades. Si bien pueden existir brechas entre el aseguramiento (cobertura poblacional) y el acceso a prestaciones de salud (cobertura prestacional efectiva) ${ }^{(7)}$, ambos se correlacionan entre sí, con asociación y repercusión sobre aspectos económicos y sociales ${ }^{(8)}$. Existen dos tipos básicos de seguro: el subsidiado y el contributivo; sustentados por el presupuesto gubernamental, y por el aporte de los empleadores o empleados; respectivamente ${ }^{(9)}$. Ello supone -más allá de la lógica diferenciación en el perfil de los afiliados- una serie de interacciones entre variables de diversa índole que puede impactar sobre el bienestar, la equidad y el acceso a los servicios de salud (SESA) ${ }^{(6)}$. Subsecuentemente, la distribución de la cobertura de aseguramiento en Salud (CAS) es crucial para el logro de la CUS.

El complejo sistema de salud peruano (SSP) no es ajeno a esta problemática. El subsector público congrega a la mayoría de los afiliados, con dos Instituciones Administradoras de Fondos de Aseguramiento en SALUD (IAFAS) principales: el Seguro Integral de Salud (SIS), con régimen básicamente subsidiado, y el Seguro Social en Salud (EsSalud), con régimen esencialmente contributivo ${ }^{(10-12)}$. En la última década aparecen dos puntos normativos claves: primero, a finales del año 2009 con la Ley Marco del Aseguramiento Universal en Salud (AUS) (13); y segundo, al finalizar el año 2013, con la reforma del sector salud (14). Posteriormente, se aprobaron normas legales para regular los procesos operativos de afiliación principalmente en el régimen subsidiado ${ }^{(15)}$. Ergo, es razonable esperar que se hayan producido cambios en la CAS y en las características de la población afiliada.

Bajo las circunstancias expuestas, si bien se han publicado estudios que exploran el avance de la CAS en Perú, principalmente del SIS, estas no incluyen el análisis del AUS posterior a la reforma ${ }^{(15)}$. En ese sentido, el objetivo del presente estudio fue describir la evolución de la CAS en Perú, para el periodo 2009-2017 y evaluar los principales factores demográficos, sociales

\section{MENSAJES CLAVE}

Motivación para realizar el estudio. En Perú, el 2009 se promulgó la Ley de Aseguramiento Universal en Salud y el 2013 se implementó la reforma del sector salud. En ese contexto, resulta relevante describir la evolución de la cobertura de aseguramiento en salud (CAS).

Principales hallazgos. La CAS global creció significativamente durante el periodo 2009-2017. Sin embargo, este crecimiento difiere entre el Seguro Integral de Salud-SIS (fundamentalmente subsidiado) y el Seguro Social en Salud-EsSalud (esencialmente contributivo), según características de la población y etapas anuales específicas.

Implicancias. Existen brechas en la CAS. Se requieren políticas públicas dirigidas a poblaciones no aseguradas.

y económicos, asociados. Nuestros resultados pueden orientar la toma de decisiones en el SSP.

\section{MATERIALES Y MÉTODOS}

\section{DISEÑO, UBICACIÓN TEMPORAL Y SELECCIÓN DE PARTICIPANTES}

Realizamos un análisis secundario de los cortes anuales repetidos de la Encuesta Nacional de Hogares (ENAHO) para el periodo 2009-2017. La ENAHO es llevada a cabo por el Instituto Nacional de Estadística e Informática (INEI) con muestreo probabilístico, estratificado y multietápico que brinda representatividad nacional, por ámbito geográfico (urbano/rural) y para cada una de las 25 regiones políticas de Perú.

\section{VARIABLES}

\section{Variable de respuesta}

Medimos las coberturas de aseguramiento de manera dicotómica: una global definida como la afiliación en al menos un seguro de salud (el individuo pudo reportar estar asegurado en más de un seguro simultáneamente), y dos específicas: SIS y EsSalud. Para los modelos de regresión, construimos una variable con tres categorías: sin seguro, SIS y EsSalud (excluimos a las personas con exclusividad en otros seguros).

\section{Variables de exposición}

El tiempo fue categorizado en cada uno de los años evaluados, y delimitado en dos etapas: 2009-2013 y 20132017. Los puntos de quiebre fueron los años: 2009 (Ley del AUS), 2013 (reforma del sector salud) y 2017 (último reporte anual de la ENAHO). Además, incluimos el ámbito 
geográfico (rural / Lima Metropolitana / resto urbano), región política (las 25 que conforman Perú), sexo (hombre / mujer), rangos de edad ( $<5$ años / niños / adolescentes / adultos jóvenes / adultos edad mediana / adultos mayores), la pobreza monetaria (pobre / no pobre) y los quintiles de gasto per-cápita del hogar (quintil1-quintil5, menor y mayor gasto, respectivamente). También, incluimos el logro educativo (sin nivel / primaria / secundaria / técnico / universitario) en personas de 25 años o más ${ }^{(16)}$, y la condición laboral (desempleado / independiente / dependiente) en personas mayores de 14 años.

\section{ANÁLISIS ESTADÍSTICO}

Usamos el módulo para muestras complejas svy (Survey complex data-analysis) del software Stata $®$ versión 14.0 (Stata Corporation, College Station, Texas, USA) con adecuación a las especificaciones del muestreo de la ENAHO: ponderaciones por estratos, factores de expansión y efecto del diseño. Para las estimaciones que requirieron la exclusión de registros (por no reporte o análisis de submuestras) empleamos la opción para sub-poblaciones (subpop).

\section{Estimación de medidas de resumen y análisis de tendencias}

Calculamos la CAS como la proporción anual ponderada a nivel global, SIS y EsSalud. La distribución de la CAS la presentamos de modo general y de acuerdo con las categorías de las variables de exposición. Para la CAS por región, usamos métodos gráficos con los mapas políticos de Perú. Para el análisis de tendencias anuales empleamos la prueba $\mathrm{F}$ de significancia conjunta para cada categoría de las variables de exposición en tres periodos de tiempo (pft): 2009-2013, 2013-2017 y 2009-2017. Asimismo, usamos dicha prueba para valorar la distribución de las variables en cada uno de los años críticos (pfd): 2009, 2013 y 2017.

\section{Modelos de regresión}

Dada la naturaleza multicategórica de la variable de respuesta, aplicamos modelos logísticos multinomiales en los años 2009, 2013 y 2017. Definimos la categoría "sin seguro" como base, frente a ser afiliado al SIS o afiliado a EsSalud. Excluimos las observaciones que registraron cobertura exclusiva por otras IAFAS (alrededor de 4,5\% de la población total). La variable logro educativo no fue incluida en los modelos ajustados debido a su colinealidad (teórica y empírica) con la edad. Debido a la alta colinealidad entre el gasto per cápita del hogar y la pobreza monetaria, cada variable fue evaluada en distintos modelos. El modelo base incluyó al gasto per cápita del hogar y la pobreza monetaria fue una prueba de sensibilidad (Anexo 1). En todos los modelos de múltiples variables ajustamos adicionalmente por la región política y la condición laboral del jefe del hogar. Calculamos razones relativas de prevalencia (RRP) con IC95\%, esta medida de asociación condicionada estima la probabilidad de tener un tipo de seguro de salud (SIS o EsSalud), frente a no tenerlo, al estar en una determinada categoría de la variable de exposición (por ejemplo: mujer) comparada con la categoría base de la exposición (por ejemplo: varón). La interpretación de las RRP es similar a cualquier medida de asociación de razón, donde el valor nulo es $1^{(17)}$.

\section{ASPECTOS ÉTICOS}

La base de la ENAHO es pública sin acceso a datos confidenciales (http://iinei.inei.gob.pe/microdatos/).

\section{RESULTADOS}

\section{DESCRIPCIÓN GENERAL DE LA COBERTURA DE ASEGURAMIENTO GLOBAL, SIS Y ESSALUD}

Observamos un aumento de la CAS global (2009: 60,5\% a 2017: $76,4 \%$ ), con incremento cercano a cinco puntos porcentuales (PP) en la etapa 2009-2013, y 11 PP en la etapa 2013-2017. Este patrón de expansión se expresa a a partir de un crecimiento anual sostenido, con excepción del 2012, donde encontramos una disminución con respecto al 2011(Figura 1A). Notamos una tendencia creciente estadísticamente significativa en todo el periodo y en las dos etapas $(p<0,001)$ (Tabla 1$)$.

En el SIS, evidenciamos un aumento de $34 \%$ a $47 \%$ para todo el periodo, con un incremento de 1,5 PP en la etapa 2009-2013 y de 11,5 PP en la etapa 2013-2019. También, percibimos la disminución de la proporción de afiliados en el 2012 con respecto al 2011 (Figura 1A). Hubo un crecimiento estadísticamente significativo durante el periodo y en cada una de las etapas $(p<0,001)$ (Tabla 2). Por su parte, en EsSalud la cobertura se elevó en la etapa 2009-2013 de $22,8 \%$ a $26,4 \%$ (Figura $1 \mathrm{~A}$ y Tabla 3 ).

\section{VARIACIONES REGIONALES EN LAS CAS GLOBAL, SIS Y ESSALUD}

En la CAS global, observamos que para el 2009, ninguna de las 25 regiones alcanzaba el $85 \%$, y 20 de ellas no superaban el 70\%. En cambio, al 2017 evidenciamos que seis y cuatro regiones alcanzaron estas coberturas, respectivamente (Figura 1B). En el caso del SIS observamos que, en el 2009, ninguna región superaba el $75 \%$ y seis tenían cobertura menor de $25 \%$, mientras que en el 2017 se evidenció que tres y ninguna región tuvieron dichas condiciones (Figura 1C). En EsSalud, notamos que solo cuatro regiones (Lima, Ica, Arequipa y Moquegua) presentaron durante el periodo de estudio una cobertura superior al 30\%. Madre de Dios tuvo una cobertura mayor a $20 \%$ hasta el 2013, luego decreció. En Ayacucho la cobertura fue inferior a $10 \%$ en el 2009, el 2013 superó dicho umbral, para retroceder el 2017(Figura 1D). 


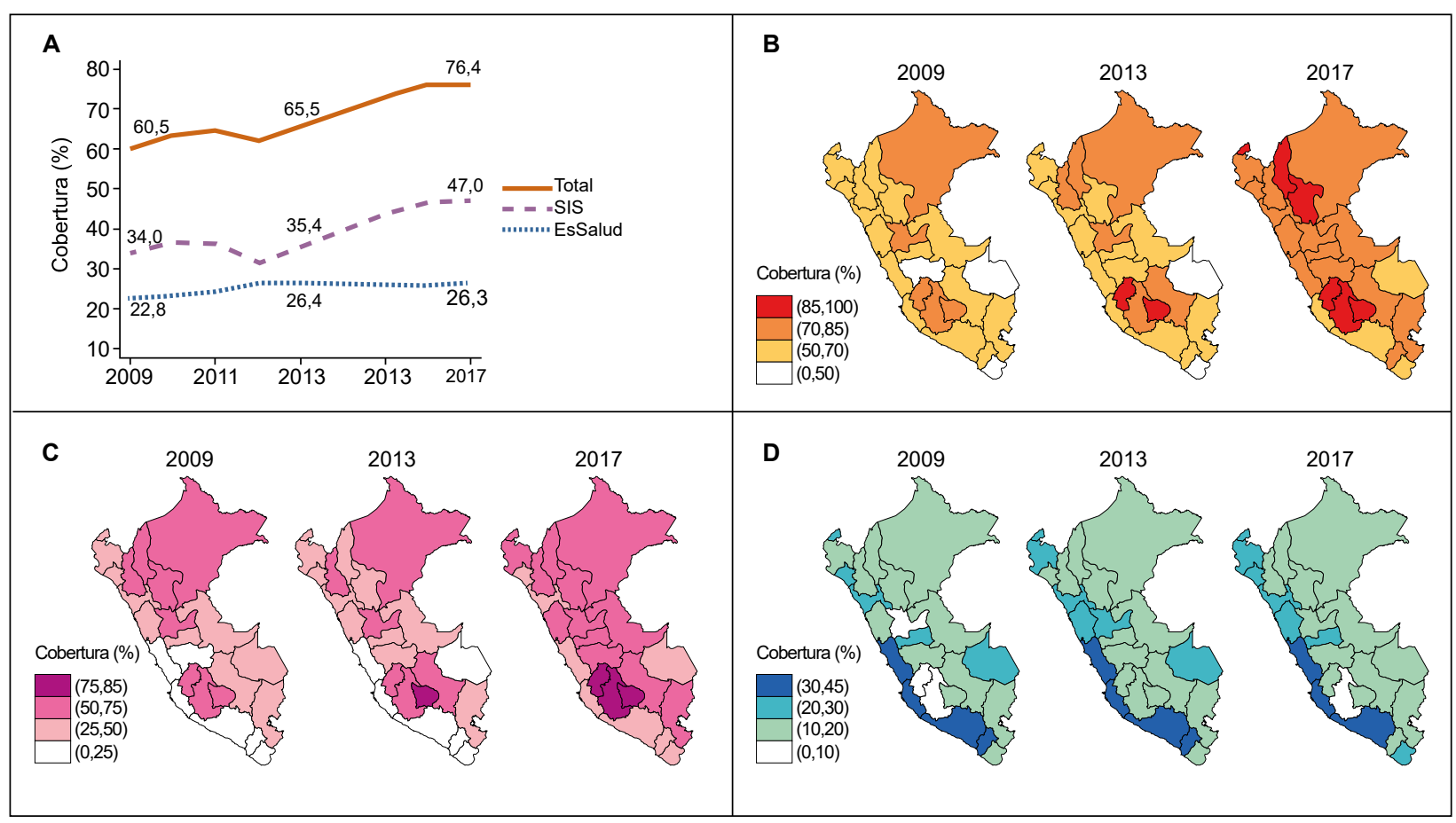

Figura 1. Cobertura de aseguramiento nacional y regional, 2009-2017. A. Cobertura de aseguramiento según tipo de seguro. B. Cobertura de aseguramiento regional global. C. Cobertura de aseguramiento regional del Seguro Integral de Salud. D. Cobertura de aseguramiento regional de EsSalud.

\section{DISTRIBUCIÓNDELACAS GLOBAL, SIS YESSALUDDE ACUERDO CON OTRAS VARIABLES DE EXPOSICIÓN}

Para el 2017 la CAS global fue mayor en: las mujeres $(78,9 \%)$, en los menores de cinco años $(86,5 \%)$, en el ámbito rural $(84 \%)$, de los quintiles de gasto 1 y $5(80 \%$ y $72,4 \%$; respectivamente), en situación de pobreza $(82,3 \%)$, en aquellos con educación superior ( 77,1 y $81,8 \%$, superior no universitaria y universitaria, respectivamente) y en trabajadores dependientes $(76,6 \%)$. Además, las variaciones durante el periodo fueron superiores en las personas: mayores de 65 años $(21,2 \mathrm{PP})$, que residen en Lima Metropolitana (20,7 PP), sin nivel educativo (24,1 PP), con educación primaria (23,5 PP) y que son trabajadores independientes (21,8 PP) (Tabla 1).

En el SIS, para el 2017, la cobertura fue superior en las mujeres $(49,7 \%)$, en los menores de cinco años $(61,5 \%)$, del en el ámbito rural $(77,8 \%)$, en el quintil de gasto $1(77,3 \%)$, en situación de pobreza $(74,7 \%)$, sin nivel educativo $(67,7 \%)$ y desempleados $(40,3 \%)$. Asimismo, las variaciones sobre el periodo fueron superiores en las personas: mayores de 65 años (20,1 PP), sin nivel educativo (23,7 PP), con educación primaria (25,6 PP) y en los trabajadores independientes (21,1 PP) (Tabla 2).

En EsSalud, en el 2017, la cobertura fue mayor en las mujeres $(26,2 \%)$, en los mayores de 65 años $(37,3 \%)$, en los que residían en Lima Metropolitana $(41,2 \%)$, del quintil $5(46,7 \%)$, en situación de no pobreza $(31,6 \%)$, en aquellos con educación superior (54,4 y $67,7 \%$, para superior no universitaria y universitaria, respectivamente) y en trabajadores dependientes (46\%). La mayor variación durante el periodo se observó en el grupo etario entre 6 y 11 años (6,1 PP) (Tabla 3).

En general, apreciamos que la CAS global creció significativamente durante todo el periodo y dentro de cada una de las dos etapas, para la mayoría de las variables de exposición, con escasas excepciones como en la educación superior universitaria (Tabla 1). En el SIS observamos que la progresión de la cobertura fue significativa en todo el periodo para la mayoría de las variables; sin embargo, este crecimiento se debe principalmente a la etapa 20132017 (Tabla 2). En EsSalud el aumento de la cobertura fue variado en todo el periodo para la mayoría de las variables; no obstante, las tendencias de crecimiento más significativas fueron en la etapa 2009-2013, en el periodo posterior en su mayoría no hubo variaciones significativas (Tabla 3).

\section{MODELOS DE REGRESIÓN LOGÍSTICOS MULTINOMIALES CRUDOS Y AJUSTADOS}

En el modelo multinomial crudo, las mujeres tuvieron mayor posibilidad, con respecto a los varones, de estar aseguradas al SIS (RRP=2009: 1,34 y 2017: 1,4) y EsSalud (RRP=2009: 1,08 y 2017: 1,22) frente a no tener seguro. Todos los grupos de edad tuvieron menor probabilidad, en comparación a los menores de cinco años, de estar afiliados al SIS o EsSalud frente a no tener seguro; excepto en los mayores de 65 años en EsSalud y con disminución 
Tabla 1. Cobertura poblacional global de aseguramiento en Perú, 2009-2017

\begin{tabular}{|c|c|c|c|c|c|c|c|c|c|c|c|c|c|c|c|}
\hline \multirow[b]{2}{*}{ Variables } & \multirow{2}{*}{$\begin{array}{c}2009 \\
\text { CP (IC 95\%) }\end{array}$} & \multicolumn{3}{|c|}{201020112012} & \multirow{2}{*}{$\begin{array}{c}2013 \\
\text { CP (IC 95\%) }\end{array}$} & \multirow{2}{*}{$\frac{2014}{C P}$} & \multicolumn{2}{|c|}{20152016} & \multirow{2}{*}{$\begin{array}{c}2017 \\
\text { CP (IC 95\%) }\end{array}$} & \multicolumn{6}{|c|}{ Diferencia porcentual en CP } \\
\hline & & CP & CP & $\mathrm{CP}$ & & & $\mathrm{CP}$ & CP & & $\begin{array}{l}2009- \\
2013\end{array}$ & pft & $\begin{array}{l}2013- \\
2017\end{array}$ & pft & $\begin{array}{l}\text { 2009- } \\
2017\end{array}$ & pft \\
\hline Población general & $60,5(59,65-61,42)$ & 63,5 & 64,5 & 61,9 & $65,5(64,75-66,15)$ & 69,0 & 73,0 & 75,8 & $76,4(75,82-77,00)$ & 4,9 & $<0,001$ & 11,0 & $<0,001$ & 15,9 & $<0,001$ \\
\hline \multicolumn{16}{|l|}{ Sexo } \\
\hline Varón & $58,3(57,30-59,20)$ & 61,3 & 62,3 & 59,9 & $63,3(62,54-64,09)$ & 66,7 & 70,3 & 73,0 & $73,8(73,15-74,51)$ & 5,1 & $<0,001$ & 10,5 & $<0,001$ & 15,6 & $<0,001$ \\
\hline Mujer & $62,8(61,77-63,74)$ & 65,6 & 66,6 & 63,8 & $67,5(66,77-68,29)$ & 71,3 & 75,5 & 78,4 & $78,9(78,23-79,51)$ & 4,8 & $<0,001$ & 11,3 & $<0,001$ & 16,1 & $<0,001$ \\
\hline pfd & $<0,001$ & & & & $<0,001$ & & & & $<0,001$ & & & & & & \\
\hline \multicolumn{16}{|l|}{ Grupo etario (años) } \\
\hline$\leq 5$ & $78,2(76,71-79,56)$ & 76,3 & 75,8 & 68,4 & $73,5(72,20-74,70)$ & 76,3 & 80,9 & 85,3 & $86,5(85,46-87,56)$ & $-4,7$ & $<0,001$ & 13,1 & $<0,001$ & 8,4 & $<0,001$ \\
\hline $06-11$ & $75,8(74,24-77,29)$ & 76,5 & 76,6 & 71,4 & $74,0(72,62-75,24)$ & 77,9 & 81,5 & 84,1 & $84,7(83,69-85,70)$ & $-1,9$ & $<0,001$ & 10,8 & $<0,001$ & 8,9 & $<0,001$ \\
\hline $12-17$ & $68,7(67,18-70,11)$ & 69,4 & 69,9 & 67,3 & $70,0(68,73-71,22)$ & 73,3 & 77,5 & 80,9 & $81,9(80,75-82,94)$ & 1,3 & 0,004 & 11,9 & $<0,001$ & 13,2 & $<0,001$ \\
\hline $18-39$ & $48,6(47,57-49,67)$ & 53,3 & 54,9 & 52,7 & $57,4(56,48-58,22)$ & 60,3 & 64,7 & 67,6 & $67,8(66,93-68,62)$ & 8,7 & $<0,001$ & 10,4 & $<0,001$ & 19,2 & $<0,001$ \\
\hline $40-64$ & $56,1(54,89-57,36)$ & 60,2 & 62,2 & 61,0 & $63,6(62,63-64,52)$ & 67,7 & 71,1 & 73,5 & $73,8(72,94-74,62)$ & 7,5 & $<0,001$ & 10,2 & $<0,001$ & 17,7 & $<0,001$ \\
\hline$\geq 65$ & $64,2(62,34-65,96)$ & 69,4 & 69,5 & 70,4 & $74,2(72,88-75,37)$ & 78,6 & 81,9 & 83,8 & $85,4(84,42-86,29)$ & 10,0 & $<0,001$ & 11,2 & $<0,001$ & 21,2 & $<0,001$ \\
\hline pfd & $<0,001$ & & & & $<0,001$ & & & & $<0,001$ & & & & & & \\
\hline \multicolumn{16}{|l|}{ Ambito geográfico } \\
\hline Rural & $71,1(69,70-72,54)$ & 75,9 & 78,4 & 71,8 & $75,1(73,94-76,16)$ & 77,7 & 81,6 & 83,4 & $84,0(83,14-84,81)$ & 3,9 & $<0,001$ & 8,9 & $<0,001$ & 12,9 & $<0,001$ \\
\hline Resto urbano & $59(57,95-60,11)$ & 62,1 & 61,8 & 58,1 & $61,7(60,79-62,67)$ & 65,3 & 70,2 & 73,6 & $74,4(73,56-75,12)$ & 2,8 & $<0,001$ & 12,6 & $<0,001$ & 15,4 & $<0,001$ \\
\hline Lima Metropolitana & $53,4(51,50-55,26)$ & 54,8 & 56,5 & 59,1 & $63,1(61,65-64,58)$ & 67,5 & 70,5 & 73,3 & $74,0(72,72-75,32)$ & 9,8 & $<0,001$ & 10,9 & $<0,001$ & 20,7 & $<0,001$ \\
\hline pfd & $<0,001$ & & & & $<0,001$ & & & & $<0,001$ & & & & & & \\
\hline \multicolumn{16}{|l|}{ Gasto per cápita por hogar } \\
\hline Quintil 1 (inferior) & $75,1(73,34-76,77)$ & 79,5 & 80,9 & 73,7 & $77,1(75,68-78,43)$ & 79,8 & 83,2 & 84,9 & $85,6(84,57-86,65)$ & 2,0 & 0,413 & 8,6 & $<0,001$ & 10,5 & $<0,001$ \\
\hline Quintil 2 & $64,7(63,03-66,27)$ & 68,8 & 68,1 & 61,1 & $67,3(65,85-68,76)$ & 71,3 & 77,0 & 79,3 & $80,3(79,15-81,42)$ & 2,7 & 0,349 & 13,0 & $<0,001$ & 15,7 & $<0,001$ \\
\hline Quintil 3 & $55,3(53,59-56,93)$ & 57,2 & 59,3 & 54,7 & $59,6(58,11-61,06)$ & 63,5 & 69,7 & 73,0 & $73,9(72,63-75,20)$ & 4,3 & 0,017 & 14,4 & $<0,001$ & 18,7 & $<0,001$ \\
\hline Quintil 4 & $50,8(49,26-52,42)$ & 54,0 & 56,4 & 55,5 & $58,7(57,42-60,00)$ & 63,8 & 66,9 & 70,6 & $70,5(69,20-71,71)$ & 7,9 & $<0,001$ & 11,8 & $<0,001$ & 19,6 & $<0,001$ \\
\hline Quintil 5 (superior) & $62,4(60,87-63,86)$ & 63,9 & 63,6 & 66,5 & $68,3(67,07-69,42)$ & 70,5 & 72,5 & 74,8 & $75,5(74,40-76,51)$ & 5,9 & $<0,001$ & 7,2 & $<0,001$ & 13,1 & $<0,001$ \\
\hline pfd & $<0,001$ & & & & $<0,001$ & & & & $<0,001$ & & & & & & \\
\hline \multicolumn{16}{|l|}{ Pobreza monetaria } \\
\hline Pobre & $66,6(65,15-67,94)$ & 70,1 & 71,5 & 65,3 & $71,6(70,28-72,89)$ & 75,3 & 79,4 & 81,5 & $82,3(81,19-83,38)$ & 5,0 & $<0,001$ & 10,7 & $<0,001$ & 15,8 & $<0,001$ \\
\hline No pobre & $57,5(56,50-58,50)$ & 60,5 & 61,8 & 60,7 & $63,5(62,77-64,27)$ & 67,2 & 71,2 & 74,3 & $74,8(74,13-75,42)$ & 6,0 & $<0,001$ & 11,3 & $<0,001$ & 17,3 & $<0,001$ \\
\hline pfd & $<0,001$ & & & & & & & & $<0,001$ & & & & & & \\
\hline \multicolumn{16}{|l|}{ Logro educativo* } \\
\hline Sin nivel & $56,2(54,62-57,66)$ & 63,3 & 65,3 & 62,6 & $66,8(65,64-68,00)$ & 72,6 & 76,9 & 78,9 & $80,2(79,23-81,13)$ & 10,7 & $<0,001$ & 13,4 & $<0,001$ & 24,1 & $<0,001$ \\
\hline Primaria & $50,4(48,88-51,84)$ & 56,9 & 57,9 & 56,6 & $60,8(59,62-62,05)$ & 66,2 & 70,1 & 73,3 & $73,9(72,78-74,97)$ & 10,5 & $<0,001$ & 13,1 & $<0,001$ & 23,5 & $<0,001$ \\
\hline Secundaria & $49,8(48,41-51,14)$ & 54,1 & 56,0 & 55,7 & $59,1(57,91-60,26)$ & 62,2 & 66,3 & 68,7 & $69,4(68,39-70,40)$ & 9,3 & $<0,001$ & 10,3 & $<0,001$ & 19,6 & $<0,001$ \\
\hline Superior no universitaria & $64,6(62,37-66,70)$ & 66,8 & 69,7 & 69,3 & $71,4(69,69-73,03)$ & 75,2 & 75,8 & 77,4 & $77,1(75,51-78,60)$ & 6,8 & $<0,001$ & 5,7 & $<0,001$ & 12,5 & $<0,001$ \\
\hline Superior universitaria & $77,1(75,06-78,92)$ & 75,8 & 77,8 & 78,2 & $79,2(77,76-80,58)$ & 80,8 & 81,6 & 82,0 & $81,8(80,32-83,16)$ & 2,2 & 0,076 & 2,6 & 0,056 & 4,7 & $<0,001$ \\
\hline pfd & $<0,001$ & & & & $<0,001$ & & & & $<0,001$ & & & & & & \\
\hline \multicolumn{16}{|l|}{ Condición laboral** } \\
\hline Desempleo & $43,6(41,15-46,07)$ & 51,0 & 50,4 & 49,6 & $52,8(50,36-55,18)$ & 53,1 & 57,8 & 63,6 & $62,2(59,69-64,70)$ & 9,2 & $<0,001$ & 9,5 & $<0,001$ & 18,6 & $<0,001$ \\
\hline Independiente & $45,2(43,98-46,49)$ & 51,2 & 52,7 & 50,0 & $54,6(53,53-55,62)$ & 59,0 & 64,0 & 66,8 & $67,1(66,10-68,01)$ & 9,3 & $<0,001$ & 12,5 & $<0,001$ & 21,8 & $<0,001$ \\
\hline Dependiente & $61,2(59,96-62,43)$ & 64,0 & 65,9 & 65,6 & $68,2(67,21-69,17)$ & 71,5 & 73,8 & 76,4 & $76,6(75,70-77,39)$ & 7,0 & $<0,001$ & 8,4 & $<0,001$ & 15,4 & $<0,001$ \\
\hline pfd & $<0,001$ & & & & $<0,001$ & & & & $<0,001$ & & & & & & \\
\hline
\end{tabular}

CP: cobertura poblacional; pft: valor de p de la prueba F de significancia conjunta para la tendencia anual de la categorías de la variable de exposición en cada periodo (2009-2013,2013-2017, 2009-2017); pfd: valor de p de la prueba F de significancia conjunta para la distribución de la variable en cada uno de los tres años críticos (2009, 2013 y 2017).

* Solo para personas de 25 años a más. * ${ }^{*}$ Solo para personas de 14 años a más.

en las personas de la tercera edad para el caso del SIS (RRP=2009: 0,27 y 2017: 0,68). La residencia en Lima Metropolitana y resto urbano tuvo menor posibilidad de aseguramiento al SIS - frente a no tener seguro - que los de la zona rural, aunque las diferencias se acortaron durante el periodo esencialmente en Lima Metropolitana $(R R P=2009$ : 0,11 y 2017: 0,22). Hubo un gradiente negativo de tener SIS y positivo de estar afiliado a EsSalud, en ambos casos frente a no tener seguro, conforme se incrementa el quintil de gasto. No obstante, dichas diferencias se han reducido en el tiempo para el quintil 5 en el SIS (RRP=2009: 0,13 y 2017: 0,19) y en EsSalud (RRP=2009: 8,32 y 2017: 5,87). Observamos no ser pobre, reduce y aumenta la posibilidad de estar afiliado al SIS y EsSalud, respectivamente, en comparación de no tener seguro; aunque las diferencias con ser pobre han incrementado ligeramente para el SIS (RRP=2009: 0,27 a 2017: 0,37) y disminuido para EsSalud ( $R R P=2009: 4,08$ a 2017: 2,87). En las personas de 25 años o más, existió un gradiente negativo de la RRP para 
Tabla 2. Cobertura poblacional de aseguramiento en el Seguro Integral de Salud para Perú, 2009-2017

\begin{tabular}{|c|c|c|c|c|c|c|c|c|c|}
\hline \multirow[b]{2}{*}{ Variables } & \multirow{2}{*}{$\begin{array}{c}2009 \\
\text { CP (IC 95\%) }\end{array}$} & \multirow{2}{*}{$\begin{array}{c}2013 \\
\text { CP (IC 95\%) }\end{array}$} & \multirow{2}{*}{$\begin{array}{c}2017 \\
\text { CP (IC 95\%) }\end{array}$} & \multicolumn{6}{|c|}{ Diferencia porcentual en CP } \\
\hline & & & & $\begin{array}{l}2009- \\
2013\end{array}$ & pft & $\begin{array}{l}2013- \\
2017\end{array}$ & pft & $\begin{array}{l}2009- \\
2017\end{array}$ & pft \\
\hline Población con SIS & $34,0(32,83-35,12)$ & $35,4(34,50-36,36)$ & $47,0(46,02-47,89)$ & 1,5 & $<0,001$ & 11,5 & $<0,001$ & 13,0 & $<0,001$ \\
\hline \multicolumn{10}{|l|}{ Sexo } \\
\hline Varón & $30,9(29,82-32,08)$ & $32,9(31,97-33,87)$ & $44,1(43,09-45,04)$ & 2,0 & $<0,001$ & 11,2 & $<0,001$ & 13,1 & $<0,001$ \\
\hline Mujer & $36,9(35,66-38,18)$ & $37,9(36,87-38,87)$ & $49,7(48,69-50,72)$ & 1,0 & $<0,001$ & 11,8 & $<0,001$ & 12,8 & $<0,001$ \\
\hline pfd & $<0,001$ & $<0,001$ & $<0,001$ & & & & & & \\
\hline \multicolumn{10}{|l|}{ Grupo etario (años) } \\
\hline$\leq 5$ & $57,5(55,67-59,30)$ & $47,7(46,19-49,17)$ & $61,5(59,90-62,97)$ & $-9,8$ & $<0,001$ & 13,8 & $<0,001$ & 4,0 & $<0,001$ \\
\hline $06-11$ & $54,7(52,88-56,53)$ & $48,7(47,16-50,23)$ & $58,3(56,75-59,75)$ & $-6,0$ & $<0,001$ & 9,6 & $<0,001$ & 3,6 & $<0,001$ \\
\hline $12-17$ & $47,5(45,82-49,16)$ & $45,3(43,81-46,70)$ & $57,0(55,47-58,52)$ & $-2,2$ & $<0,001$ & 11,8 & $<0,001$ & 9,5 & $<0,001$ \\
\hline $18-39$ & $24,5(23,40-25,54)$ & $29,2(28,25-30,11)$ & $41,6(40,52-42,62)$ & 4,7 & $<0,001$ & 12,4 & $<0,001$ & 17,1 & $<0,001$ \\
\hline $40-64$ & $22,3(21,21-23,44)$ & $29,3(28,35-30,33)$ & $40,3(39,23-41,34)$ & 7,0 & $<0,001$ & 11,0 & $<0,001$ & 18,0 & $<0,001$ \\
\hline$\geq 65$ & $25,0(23,23-26,95)$ & $34,0(32,43-35,55)$ & $45,1(43,56-46,72)$ & 8,9 & $<0,001$ & 11,2 & $<0,001$ & 20,1 & $<0,001$ \\
\hline pfd & $<0,001$ & $<0,001$ & $<0,001$ & & & & & & \\
\hline \multicolumn{10}{|l|}{ Ambito geográfico } \\
\hline Rural & $66,0(64,40-67,56)$ & $68,6(67,31-69,92)$ & $77,8(76,76-78,87)$ & 2,6 & $<0,001$ & 9,2 & $<0,001$ & 11,8 & $<0,001$ \\
\hline Resto urbano & $29,7(28,27-31,12)$ & $29,9(28,79-31,02)$ & $44,9(43,72-45,99)$ & 0,2 & $<0,001$ & 15,0 & $<0,001$ & 15,2 & $<0,001$ \\
\hline Lima Metropolitana & $11,8(10,60-13,10)$ & $17,1(15,75-18,59)$ & $28,3(26,48-30,19)$ & 5,3 & $<0,001$ & 11,2 & $<0,001$ & 16,5 & $<0,001$ \\
\hline pfd & $<0,001$ & $<0,001$ & $<0,001$ & & & & & & \\
\hline \multicolumn{10}{|l|}{ Gasto per cápita por hogar } \\
\hline Quintil 1 (inferior) & $74,1(72,32-75,81)$ & $74,7(73,22-76,19)$ & $83,2(82,02-84,31)$ & 0,6 & $<0,001$ & 8,5 & $<0,001$ & 9,1 & $<0,001$ \\
\hline Quintil 2 & $58,4(56,63-60,15)$ & $56,9(55,27-58,50)$ & $69,3(67,78-70,75)$ & $-1,5$ & $<0,001$ & 12,4 & $<0,001$ & 10,9 & $<0,001$ \\
\hline Quintil 3 & $38,4(36,56-40,17)$ & $36,9(35,38-38,42)$ & $51,1(49,47-52,69)$ & $-1,5$ & $<0,001$ & 14,2 & $<0,001$ & 12,7 & $<0,001$ \\
\hline Quintil 4 & $19,5(18,27-20,87)$ & $21,1(19,95-22,29)$ & $34,4(32,95-35,78)$ & 1,6 & $<0,001$ & 13,3 & $<0,001$ & 14,8 & $<0,001$ \\
\hline Quintil 5 (superior) & $6,5(5,85-7,19)$ & $8,5(7,78-9,28)$ & $15,6(14,61-16,65)$ & 2,0 & $<0,001$ & 7,1 & $<0,001$ & 9,1 & $<0,001$ \\
\hline pfd & $<0,001$ & $<0,001$ & $<0,001$ & & & & & & \\
\hline \multicolumn{10}{|l|}{ Pobreza monetaria } \\
\hline Pobre & $60,4(58,80-62,01)$ & $64,2(62,63-65,76)$ & $74,7(73,16-76,13)$ & 3,8 & $<0,001$ & 10,5 & $<0,001$ & 14,3 & $<0,001$ \\
\hline No pobre & $20,6(19,71-21,58)$ & $26,4(25,54-27,22)$ & $39,3(38,35-40,20)$ & 5,7 & $<0,001$ & 12,9 & $<0,001$ & 18,6 & $<0,001$ \\
\hline pfd & $<0,001$ & $<0,001$ & $<0,001$ & & & & & & \\
\hline \multicolumn{10}{|l|}{ Logro educativo* } \\
\hline Sin nivel & $44,1(42,39-45,78)$ & $53,7(52,32-55,08)$ & $67,7(66,49-68,95)$ & 9,6 & $<0,001$ & 14,0 & $<0,001$ & 23,7 & $<0,001$ \\
\hline Primaria & $29,3(27,88-30,70)$ & $39,3(38,02-40,52)$ & $54,9(53,60-56,17)$ & 10,0 & $<0,001$ & 15,6 & $<0,001$ & 25,6 & $<0,001$ \\
\hline Secundaria & $14,2(13,21-15,17)$ & $19,6(18,66-20,53)$ & $31,5(30,34-32,58)$ & 5,4 & $<0,001$ & 11,9 & $<0,001$ & 17,3 & $<0,001$ \\
\hline Superior no universitaria & $8,1(7,06-9,29)$ & $11(9,91-12,29)$ & $17,8(16,37-19,33)$ & 2,9 & $<0,001$ & 6,8 & $<0,001$ & 9,7 & $<0,001$ \\
\hline Superior universitaria & $1,8(1,34-2,27)$ & $3,1(2,56-3,68)$ & $5,9(5,19-6,78)$ & 1,3 & 0,008 & 2,9 & $<0,001$ & 4,2 & $<0,001$ \\
\hline pfd & $<0,001$ & $<0,001$ & $<0,001$ & & & & & & \\
\hline \multicolumn{10}{|l|}{ Condición laboral ${ }^{* *}$} \\
\hline Desempleo & $22,9(20,73-25,23)$ & $29,8(27,64-32,13)$ & $40,3(37,61-42,97)$ & 6,9 & $<0,001$ & 10,4 & $<0,001$ & 17,4 & $<0,001$ \\
\hline Independiente & $31,3(30,06-32,61)$ & $39,6(38,51-40,70)$ & $52,4(51,35-53,53)$ & 8,3 & $<0,001$ & 12,8 & $<0,001$ & 21,1 & $<0,001$ \\
\hline Dependiente & $14,4(13,48-15,35)$ & $18,2(17,38-19,00)$ & $26,5(25,51-27,57)$ & 3,8 & $<0,001$ & 8,4 & $<0,001$ & 12,1 & $<0,001$ \\
\hline pfd & $<0,001$ & $<0,001$ & $<0,001$ & & & & & & \\
\hline
\end{tabular}

CP: cobertura poblacional; SIS: Seguro Integral de Salud; pft: valor de $\mathrm{p}$ de la prueba $\mathrm{F}$ de significancia conjunta para la tendencia anual de la categorias de la variable de exposición en cada periodo (2009-2013,2013-2017, 2009-2017); pfd: valor de $p$ de la prueba F de significancia conjunta para la distribución de la variable en cada uno de los tres años críticos (2009, 2013 y 2017).

* Solo para personas de 25 años a más. ** Solo para personas de 14 años a más. 
Tabla 3. Cobertura poblacional de aseguramiento en EsSalud para Perú, 2009-2017

\begin{tabular}{|c|c|c|c|c|c|c|c|c|c|}
\hline \multirow[b]{2}{*}{ Variables } & \multirow{2}{*}{$\begin{array}{c}2009 \\
\text { CP (IC 95\%) }\end{array}$} & \multirow{2}{*}{$\begin{array}{c}2013 \\
\text { CP (IC 95\%) }\end{array}$} & \multirow{2}{*}{$\begin{array}{c}2017 \\
\text { CP (IC 95\%) }\end{array}$} & \multicolumn{6}{|c|}{ Diferencia porcentual en CP } \\
\hline & & & & $\begin{array}{l}2009- \\
2013 \\
\end{array}$ & pft & $\begin{array}{l}2013- \\
2017 \\
\end{array}$ & pft & $\begin{array}{l}2009- \\
2017\end{array}$ & pft \\
\hline Población con EsSalud & $22,8(21,96-23,69)$ & $26,4(25,66-27,19)$ & $26,3(25,57-27,11)$ & 3,6 & $<0,001$ & $-0,1$ & 0,841 & 3,5 & $<0,001$ \\
\hline \multicolumn{10}{|l|}{ Sexo } \\
\hline Varón & $23,2(22,29-24,13)$ & $26,3(25,56-27,15)$ & $26,5(25,68-27,32)$ & 3,1 & $<0,001$ & 0,1 & 0,825 & 3,3 & $<0,001$ \\
\hline Mujer & $22,4(21,54-23,37)$ & $26,5(25,67-27,33)$ & $26,2(25,35-27,03)$ & 4,1 & $<0,001$ & $-0,3$ & 0,811 & 3,7 & $<0,001$ \\
\hline pfd & 0,016 & 0,606 & 0,312 & & & & & & \\
\hline \multicolumn{10}{|l|}{ Grupo etario (años) } \\
\hline$\leq 5$ & $19,0(17,69-20,35)$ & $24,0(22,74-25,36)$ & $23,1(21,77-24,44)$ & 5,1 & $<0,001$ & $-1,0$ & 0,725 & 4,1 & $<0,001$ \\
\hline $06-11$ & $18,8(17,54-20,10)$ & $23,3(22,02-24,53)$ & $24,9(23,62-26,22)$ & 4,5 & $<0,001$ & 1,6 & 0,432 & 6,1 & $<0,001$ \\
\hline $12-17$ & $18,4(17,27-19,67)$ & $22,1(20,89-23,28)$ & $22,4(21,18-23,67)$ & 3,6 & $<0,001$ & 0,3 & 0,705 & 4,0 & $<0,001$ \\
\hline $18-39$ & $19,2(18,33-20,13)$ & $23,3(22,45-24,14)$ & $22,1(21,28-22,93)$ & 4,1 & $<0,001$ & $-1,2$ & 0,094 & 2,9 & $<0,001$ \\
\hline $40-64$ & $29,4(28,20-30,70)$ & $30,3(29,35-31,33)$ & $30,2(29,17-31,18)$ & 0,9 & 0,003 & $-0,2$ & 0,982 & 0,7 & 0,032 \\
\hline$\geq 65$ & $35,6(33,51-37,80)$ & $36,9(35,27-38,47)$ & $37,3(35,68-38,90)$ & 1,2 & 0,086 & 0,4 & 0,588 & 1,7 & 0,210 \\
\hline pfd & $<0,001$ & $<0,001$ & $<0,001$ & & & & & & \\
\hline \multicolumn{10}{|l|}{ Ambito geográfico } \\
\hline Rural & $5,4(4,26-5,44)$ & $6,7(5,44-6,66)$ & $6,4(5,30-6,41)$ & 1,2 & 0,004 & $-0,3$ & 0,478 & 1,0 & 0,007 \\
\hline Resto urbano & $27,6(25,24-27,56)$ & $29,9(27,97-29,94)$ & $28,2(26,35-28,19)$ & 2,4 & $<0,001$ & $-1,8$ & 0,135 & 0,6 & $<0,001$ \\
\hline Lima Metropolitana & $35,6(31,83-35,55)$ & $40,5(37,25-40,54)$ & $41,2(37,61-41,17)$ & 5,0 & $<0,001$ & 0,6 & 0,524 & 5,6 & $<0,001$ \\
\hline pfd & $<0,001$ & $<0,001$ & $<0,001$ & & & & & & \\
\hline \multicolumn{10}{|l|}{ Gasto per cápita por hogar } \\
\hline Quintil 1 (inferior) & $0,9(0,63-1,19)$ & $2,3(1,75-2,88)$ & $2,4(1,98-2,86)$ & 1,4 & 0,010 & 0,1 & 0,284 & 1,5 & $<0,001$ \\
\hline Quintil 2 & $6,1(5,26-6,98)$ & $9,9(8,99-10,86)$ & $10,6(9,58-11,65)$ & 3,8 & $<0,001$ & 0,7 & 0,773 & 4,5 & $<0,001$ \\
\hline Quintil 3 & $15,9(14,62-17,18)$ & $21,2(19,96-22,53)$ & $21,9(20,55-23,29)$ & 5,3 & $<0,001$ & 0,7 & 0,809 & 6,0 & $<0,001$ \\
\hline Quintil 4 & $28,2(26,68-29,69)$ & $34,1(32,77-35,47)$ & $33,3(31,96-34,74)$ & 5,9 & $<0,001$ & $-0,8$ & 0,574 & 5,1 & $<0,001$ \\
\hline Quintil 5 (superior) & $45,5(43,87-47,06)$ & $50,0(48,65-51,31)$ & $50,6(49,15-51,97)$ & 4,5 & $<0,001$ & 0,6 & 0,496 & 5,1 & $<0,001$ \\
\hline pfd & $<0,001$ & $<0,001$ & $<0,001$ & & & & & & \\
\hline \multicolumn{10}{|l|}{ Pobreza monetaria } \\
\hline Pobre & $5,8(5,13-6,60)$ & $7,1(6,27-7,98)$ & $7,4(6,45-8,42)$ & 1,3 & 0,174 & 0,3 & 0,256 & 1,6 & 0,035 \\
\hline No pobre & $31,4(30,37-32,4)$ & $32,5(31,65-33,36)$ & $31,6(30,75-32,44)$ & 1,1 & $<0,001$ & $-0,9$ & 0,305 & 0,2 & $<0,001$ \\
\hline pfd & $<0,001$ & $<0,001$ & $<0,001$ & & & & & & \\
\hline \multicolumn{10}{|l|}{ Logro educativo* } \\
\hline Sin nivel & $11,5(10,52-12,54)$ & $12,6(11,73-13,50)$ & $11,9(11,06-12,89)$ & 1,1 & 0,251 & $-0,7$ & 0,164 & 0,4 & 0,176 \\
\hline Primaria & $19,6(18,42-20,90)$ & $20,1(19,07-21,15)$ & $18,0(16,99-19,08)$ & 0,5 & 0,327 & $-2,1$ & 0,007 & $-1,6$ & 0,016 \\
\hline Secundaria & $30,4(29,10-31,76)$ & $34,5(33,34-35,65)$ & $33,9(32,70-35,01)$ & 4,1 & $<0,001$ & $-0,6$ & 0,417 & 3,4 & $<0,001$ \\
\hline Superior no universitaria & $49,2(46,81-51,55)$ & $54,5(52,61-56,41)$ & $54,4(52,53-56,30)$ & 5,3 & $<0,001$ & $-0,1$ & 0,394 & 5,2 & $<0,001$ \\
\hline Superior universitaria & $64,9(62,49-67,21)$ & $68,0(66,30-69,69)$ & $67,7(66,03-69,41)$ & 3,1 & 0,066 & $-0,3$ & 0,380 & 2,8 & 0.034 \\
\hline pfd & $<0,001$ & $<0,001$ & $<0,001$ & & & & & & \\
\hline \multicolumn{10}{|l|}{ Condición laboral ${ }^{*}$} \\
\hline Desempleo & $16,3(14,53-18,27)$ & $17,3(15,45-19,30)$ & $17,9(15,82-20,12)$ & 1,0 & 0,426 & 0,6 & 0,866 & 1,6 & 0,774 \\
\hline Independiente & $11,9(11,18-12,66)$ & $13,0(12,34-13,66)$ & $12,7(12,11-13,40)$ & 1,1 & 0,009 & $-0,3$ & 0,872 & 0,8 & 0,091 \\
\hline Dependiente & $41,5(40,10-42,82)$ & $45,1(43,94-46,17)$ & $46,0(44,85-47,09)$ & 3,6 & $<0,001$ & 0,9 & 0,320 & 4,5 & $<0,001$ \\
\hline pfd & $<0,001$ & $<0,001$ & $<0,001$ & & & & & & \\
\hline
\end{tabular}

CP: cobertura poblacional; pft: valor de $\mathrm{p}$ de la prueba F de significancia conjunta para la tendencia anual de la categorías de la variable de exposición en cada periodo (2009-2013,20132017, 2009-2017); pfd: valor de p de la prueba F de significancia conjunta para la distribución de la variable en cada uno de los tres años críticos (2009, 2013 y 2017).

* Solo para personas de 25 años a más. ** Solo para personas de 14 años a más. 
el SIS y positivo para EsSalud conforme crece el nivel de instrucción; sin cambios significativos y con disminución a lo largo del periodo de estudio (RRP=2009: 9,82 y 2017: $5,60)$; respectivamente. En la población mayor de 14 años, hubo una mayor probabilidad de tener SIS y EsSalud, frente a no tener seguro, en los trabajadores independientes y dependientes, respectivamente; sin diferencias durante el periodo (Tabla 4).

En los modelos ajustados, las mujeres presentaron una mayor posibilidad, con respecto a los varones, de estar aseguradas al SIS (RRP=2009: 1,65 y 2017: 1,53) y EsSalud (RRP=2013: 1,11 y 2017: 1,23). Asimismo, la mayoría de los grupos de edad tuvieron menor posibilidad, en comparación a los menores de cinco años, de estar afiliados al SIS o EsSalud frente a no tener seguro; excepto en el grupo de 6 a 11 años y en los mayores de 65 años, donde no se encontraron diferencias significativas. La residencia en el resto urbano, con respecto a las zonas rurales, tuvo menor posibilidad de aseguramiento al SIS (RRP=2009: 0,48 y 2017: 0,69) y mayor posibilidad de aseguramiento a EsSalud (RRP=2009: 1,29 y 2017: $1,43)$. Finalmente, encontramos un gradiente negativo de tener SIS y positivo de estar afiliado a EsSalud, en ambos casos frente a no tener seguro, conforme se incrementa el quintil de gasto; dichas diferencias se han reducido significativamente en el tiempo para el quintil 5 en EsSalud (RRP=2009: 33,58 y 2017: 8,54) (Tabla 5). En el modelo ajustado por los quintiles de gasto observamos resultados similares (Anexo 1).

\section{DISCUSIÓN}

Nuestros hallazgos muestran un crecimiento de la CAS en el periodo, aunque con ciclos diferenciados de expansión y con disparidades en las características de la población afiliada de acuerdo con las IAFAS analizadas. En la primera etapa comprendida entre la promulgación de la ley del AUS (2009) y la reforma del sector salud (2013) hubo un mínimo crecimiento de la CAS global, mayor en EsSalud que en el SIS. Mientras que en la etapa siguiente (20132017), el incremento de la CAS global fue superior, con aumento marcado en el SIS y sin variaciones significativas en EsSalud. Esto reflejaría una tendencia de despliegue de las políticas públicas de aseguramiento que amerita un abordaje contextualizado para el SSP y diferenciado

Tabla 4. Variables asociadas a la condición de aseguramiento (modelos de regresión logística multinomial crudos*)

\begin{tabular}{|c|c|c|c|c|c|c|c|c|c|}
\hline \multirow{3}{*}{ Variables } & \multicolumn{3}{|c|}{2009} & \multicolumn{3}{|c|}{2013} & \multicolumn{3}{|c|}{2017} \\
\hline & \multicolumn{3}{|c|}{$\mathrm{N}=27681322 ; \mathrm{n}=85829$} & \multicolumn{3}{|c|}{$\mathrm{N}=29412845 ; \mathrm{n}=112496$} & \multicolumn{3}{|c|}{$\mathrm{N}=30685310 ; n=119884$} \\
\hline & NS & $\begin{array}{c}\text { SIS } \\
\operatorname{RRP}(\text { IC 95\%) }\end{array}$ & $\begin{array}{c}\text { EsSalud } \\
\text { RRP (IC 95\%) }\end{array}$ & NS & $\begin{array}{c}\text { SIS } \\
\text { RRP (IC 95\%) }\end{array}$ & $\begin{array}{c}\text { EsSalud } \\
\text { RRP (IC 95\%) }\end{array}$ & NS & $\begin{array}{c}\text { SIS } \\
\text { RRP (IC 95\%) }\end{array}$ & $\begin{array}{c}\text { EsSalud } \\
\text { RRP (IC 95\%) }\end{array}$ \\
\hline \multicolumn{10}{|l|}{ Sexo } \\
\hline Mujer / Varón & Ref. & $1,34(1,29-1,39)$ & $1,08(1,04-1,13)$ & Ref. & $1,30(1,26-1,34)$ & $1,14(1,10-1,18)$ & Ref. & $1,40(1,35-1,45)$ & $1,22(1,17-1,27)$ \\
\hline \multicolumn{10}{|l|}{ Grupo etario } \\
\hline $6-11 / \leq 5$ & Ref. & $0,86(0,79-0,94)$ & $0,90(0,80-1,01)$ & Ref. & $1,04(0,96-1,12)$ & $0,99(0,90-1,09)$ & Ref. & $0,83(0,76-0,92)$ & $0,95(0,85-1,06)$ \\
\hline $12-17 / \leq 5$ & Ref. & $0,58(0,53-0,63)$ & $0,68(0,61-0,77)$ & Ref. & $0,84(0,78-0,91)$ & $0,82(0,74-0,91)$ & Ref. & $0,69(0,62-0,77)$ & $0,72(0,64-0,81)$ \\
\hline $18-39 / \leq 5$ & Ref. & $0,18(0,17-0,20)$ & $0,44(0,40-0,48)$ & Ref. & $0,38(0,36-0,40)$ & $0,60(0,56-0,65)$ & Ref. & $0,28(0,26-0,31)$ & $0,4(0,36-0,44)$ \\
\hline $40-64 / \leq 5$ & Ref. & $0,19(0,18-0,21)$ & $0,78(0,70-0,88)$ & Ref. & $0,45(0,42-0,48)$ & $0,92(0,84-1,01)$ & Ref. & $0,34(0,31-0,37)$ & $0,67(0,59-0,75)$ \\
\hline 65 o más $/ \leq 5$ & Ref. & $0,27(0,24-0,30)$ & $1,19(1,04-1,36)$ & Ref. & $0,73(0,67-0,81)$ & $1,58(1,41-1,77)$ & Ref. & $0,68(0,60-0,76)$ & $1,50(1,31-1,72)$ \\
\hline \multicolumn{10}{|l|}{ Ámbito geográfico } \\
\hline Resto urbano / Rural & Ref. & $0,31(0,28-0,35)$ & $3,82(3,31-4,40)$ & Ref. & $0,28(0,26-0,31)$ & $3,09(2,74-3,48)$ & Ref. & $0,36(0,33-0,39)$ & $2,88(2,57-3,23)$ \\
\hline Lima Metropolitana / Rural & Ref. & $0,11(0,10-0,13)$ & $4,04(3,46-4,72)$ & Ref. & $0,17(0,15-0,19)$ & $4,07(3,57-4,63)$ & Ref. & $0,22(0,20-0,25)$ & $3,89(3,42-4,44)$ \\
\hline \multicolumn{10}{|l|}{ Gasto per cápita por hogar } \\
\hline Quintil 2/ Quintil 1 & Ref. & $0,55(0,50-0,62)$ & $5,17(3,62-7,38)$ & Ref. & $0,53(0,48-0,59)$ & $3,10(2,34-4,11)$ & Ref. & $0,61(0,55-0,68)$ & $3,24(2,61-4,01)$ \\
\hline Quintil 3/ Quintil 1 & Ref. & $0,29(0,25-0,32)$ & $10,99(7,80-15,48)$ & Ref. & $0,28(0,25-0,31)$ & $5,34(4,08-6,99)$ & Ref. & $0,34(0,30-0,38)$ & $5,05(4,07-6,25)$ \\
\hline Quintil 4 / Quintil 1 & Ref. & $0,13(0,12-0,15)$ & $17,70(12,62-24,83)$ & Ref. & $0,16(0,14-0,17)$ & $8,35(6,41-10,88)$ & Ref. & $0,20(0,18-0,22)$ & $6,72(6,72-8,27)$ \\
\hline Quintil 5/ Quintil 1 & Ref. & $0,06(0,05-0,07)$ & $34,21(24,44-47,90)$ & Ref. & $0,08(0,07-0,09)$ & $14,7(11,3-19,13)$ & Ref. & $0,11(0,10-0,12)$ & $11,40(11,4-13,98)$ \\
\hline \multicolumn{10}{|l|}{ Pobreza monetaria } \\
\hline No pobre / pobre & Ref. & $0,27(0,25-0,29)$ & $4,08(3,55-4,70)$ & Ref. & $0,32(0,30-0,34)$ & $3,42(2,99-3,91)$ & Ref. & $0,37(0,34-0,40)$ & $2,87(2,47-3,33)$ \\
\hline \multicolumn{10}{|l|}{ Logro educativo** } \\
\hline Primaria / Sin nivel & Ref. & $0,58(0,54-0,63)$ & $1,51(1,34-1,69)$ & Ref. & $0,62(0,58-0,66)$ & $1,35(1,23-1,48)$ & Ref. & $0,61(0,57-0,66)$ & $1,14(1,02-1,28)$ \\
\hline Secundaria / Sin nivel & Ref. & $0,28(0,25-0,31)$ & $2,26(2,01-2,53)$ & Ref. & $0,30(0,27-0,32)$ & $2,16(1,96-2,37)$ & Ref. & $0,30(0,28-0,32)$ & $1,79(1,60-1,99)$ \\
\hline $\begin{array}{l}\text { Superior no universitaria / } \\
\text { Sin nivel }\end{array}$ & Ref. & $0,23(0,19-0,27)$ & $5,15(4,46-5,94)$ & Ref. & $0,24(0,21-0,27)$ & $4,82(4,28-5,44)$ & Ref. & $0,23(0,20-0,26)$ & $3,82(3,36-4,34)$ \\
\hline $\begin{array}{l}\text { Superior universitaria / } \\
\text { Sin nivel }\end{array}$ & Ref. & $0,07(0,05-0,10)$ & $9,82(8,47-11,4)$ & Ref. & $0,09(0,07-0,11)$ & $7,86(6,96-8,87)$ & Ref. & $0,10(0,08-0,11)$ & $5,60(4,89-6,40)$ \\
\hline \multicolumn{10}{|l|}{ Condición laboral${ }^{\star \star \star}$} \\
\hline Independiente / Desempleado & Ref. & $1,41(1,23-1,60)$ & $0,74(0,64-0,86)$ & Ref. & $1,39(1,24-1,56)$ & $0,79(0,67-0,92)$ & Ref. & $1,50(1,32-1,69)$ & $0,84(0,71-0,99)$ \\
\hline Dependiente / Desempleado & Ref. & $0,90(0,79-1,03)$ & $3,58(3,09-4,15)$ & Ref. & $0,91(0,81-1,02)$ & $3,87(3,34-4,47)$ & Ref. & $1,06(0,94-1,20)$ & $4,20(3,56-4,95)$ \\
\hline
\end{tabular}

NS: Sin seguro de salud; SIS: Seguro Integral de Salud; RRP: razón relativa de prevalencia.

* Los modelos son independientes para cada año y se consideró el diseño muestral complejo de la encuesta.

** Solo para personas de 25 años a más. *** Solo para personas de 14 años a más. 
Tabla 5. Variables asociadas a la condición de aseguramiento (modelos de regresión logística multinomial ajustados*)

\begin{tabular}{|c|c|c|c|c|c|c|c|c|c|}
\hline \multirow{3}{*}{ Variables** } & \multicolumn{3}{|c|}{2009} & \multicolumn{3}{|c|}{2013} & \multicolumn{3}{|c|}{2017} \\
\hline & \multicolumn{3}{|c|}{$\mathrm{N}=24032$ 620; n=76 821} & \multicolumn{3}{|c|}{ N=24 786 256; n=98 161} & \multicolumn{3}{|c|}{$\mathrm{N}=26003$ 214; n=104 103} \\
\hline & NS & $\begin{array}{c}\text { SIS } \\
\text { RRP (IC 95\%) }\end{array}$ & $\begin{array}{c}\text { EsSalud } \\
\text { RRP (IC 95\%) }\end{array}$ & NS & $\begin{array}{c}\text { SIS } \\
\text { RRP (IC 95\%) }\end{array}$ & $\begin{array}{c}\text { EsSalud } \\
\text { RRP (IC 95\%) }\end{array}$ & NS & $\begin{array}{c}\text { SIS } \\
\text { RRP (IC 95\%) }\end{array}$ & $\begin{array}{c}\text { EsSalud } \\
\text { RRP (IC 95\%) }\end{array}$ \\
\hline \multicolumn{10}{|l|}{ Sexo } \\
\hline Mujer / Varón & Ref. & $1,65(1,58-1,73)$ & $1,02(0,98-1,08)$ & Ref. & $1,43(1,38-1,48)$ & $1,11(1,06-1,16)$ & Ref. & $1,53(1,47-1,60)$ & $1,23(1,17-1,29)$ \\
\hline \multicolumn{10}{|l|}{ Grupo de edad (años) } \\
\hline $6-11 / \leq 5$ & Ref. & $0,79(0,71-0,87)$ & $0,91(0,80-1,04)$ & Ref. & $0,99(0,91-1,08)$ & $1,02(0,91-1,14)$ & Ref. & $0,79(0,70-0,88)$ & $0,95(0,83-1,08)$ \\
\hline $12-17 / \leq 5$ & Ref. & $0,52(0,47-0,58)$ & $0,71(0,62-0,82)$ & Ref. & $0,79(0,73-0,87)$ & $0,85(0,76-0,96)$ & Ref. & $0,69(0,62-0,78)$ & $0,75(0,65-0,86)$ \\
\hline $18-39 / \leq 5$ & Ref. & $0,17(0,15-0,19)$ & $0,38(0,34-0,43)$ & Ref. & $0,46(0,43-0,50)$ & $0,50(0,45-0,54)$ & Ref. & $0,33(0,30-0,37)$ & $0,32(0,29-0,36)$ \\
\hline $40-64 / \leq 5$ & Ref. & $0,17(0,15-0,19)$ & $0,73(0,64-0,83)$ & Ref. & $0,53(0,49-0,58)$ & $0,82(0,74-0,91)$ & Ref. & $0,40(0,36-0,44)$ & $0,59(0,52-0,68)$ \\
\hline 65 o más $/ \leq 5$ & Ref. & $0,19(0,17-0,22)$ & $0,97(0,81-1,17)$ & Ref. & $0,75(0,66-0,84)$ & $1,16(0,99-1,36)$ & Ref. & $0,75(0,66-0,86)$ & $1,13(0,96-1,34)$ \\
\hline \multicolumn{10}{|l|}{ Ámbito geográfico } \\
\hline Resto urbano / Rural & Ref. & $0,48(0,43-0,54)$ & $1,29(1,11-1,51)$ & Ref. & $0,53(0,49-0,59)$ & $1,44(1,26-1,64)$ & Ref. & $0,69(0,62-0,75)$ & $1,43(1,26-1,62)$ \\
\hline Lima Metropolitana / Rural & Ref. & 0,27 (0,19-0,39) & $1,09(0,78-1,53)$ & Ref. & $0,40(0,31-0,52)$ & $1,02(0,79-1,32)$ & Ref. & $0,61(0,50-0,76)$ & $1,33(1,06-1,68)$ \\
\hline \multicolumn{10}{|l|}{ Pobreza monetaria } \\
\hline Quintil 2 / Quintil 1 & Ref. & $0,88(0,78-0,99)$ & $4,90(3,30-7,28)$ & Ref. & $0,77(0,69-0,86)$ & $2,38(1,76-3,22)$ & Ref. & $0,83(0,74-0,93)$ & $2,61(2,05-3,32)$ \\
\hline Quintil 3 / Quintil 1 & Ref. & $0,65(0,57-0,75)$ & $10,15(6,88-14,97)$ & Ref. & $0,53(0,47-0,59)$ & $3,86(2,89-5,17)$ & Ref. & $0,55(0,48-0,62)$ & $3,77(2,95-4,82)$ \\
\hline Quintil 4 / Quintil 1 & Ref. & $0,38(0,33-0,44)$ & $16,32(11,10-24,00)$ & Ref. & $0,32(0,28-0,37)$ & $6,07(4,54-8,11)$ & Ref. & $0,37(0,32-0,42)$ & $4,90(3,84-6,27)$ \\
\hline Quintil 5 / Quintil 1 & Ref. & $0,18(0,15-0,22)$ & $33,58(22,78-49,49)$ & Ref. & $0,18(0,15-0,21)$ & $10,95(8,19-14,64)$ & Ref. & $0,21(0,18-0,25)$ & $8,54(6,67-10,94)$ \\
\hline
\end{tabular}

NS: Sin seguro de salud; SIS: Seguro Integral de Salud; RRP: razón relativa de prevalencia.

* Los modelos son independientes para cada año y se consideró el diseño muestral complejo de la encuesta.

** Se ajustó adicionalmente por la condición laboral del jefe del hogar y por la región política.

básicamente por la naturaleza de la financiación para los regímenes subsidiado, contributivo y semi-contributivo ${ }^{(11,15)}$. Lo encontrado en las dos principales IAFAS del SSP serían una expresión de los cambios normativos a través del tiempo, sobre todo en los sectores salud y trabajo, para el SIS y EsSalud, respectivamente. Asimismo, las variaciones descritas se encontrarían estrechamente relacionados con aspectos económicos y sociales del país, donde se incluyen la informalidad laboral, la vulnerabilidad y la pobreza.

El pequeño ascenso de la CAS observado tras la promulgación de la Ley Marco del AUS ${ }^{(13)}$, parece ir en concordancia con la penetración focalizada a poblaciones rurales, en pobreza y pobreza extrema ${ }^{(15)}$. Posteriormente, se incrementa la CAS, lo cual puede explicarse por el potencial impacto de las acciones de la reforma, donde se realizan arreglos institucionales para la extensión de la cobertura en un marco de los DES y se articula con intervenciones de protección social ${ }^{(14,18)}$. Adicionalmente, se aprobaron normas que incluyen afiliación directa al régimen subsidiado para poblaciones vulnerables, grupos con protección especial (como los estudiantes de educación básica regular, beneficiarios de programas sociales, entre otros) y acceso al régimen semi-contributivo para trabajadores independientes ${ }^{(14,15)}$. Lo expuesto podría asociarse con la elevación relativa de población no pobre, adulta mayor (pensión 65), con trabajo y con quintiles de gastos superiores esta IAFAS observado principalmente en la segunda etapa.

Debemos remarcar que, en el proceso de afiliación subsidiada regular del SIS, el Sistema de Focalización de Hogares (SISFOH) del Ministerio de Inclusión Social juega un rol preponderante al asignar una clasificación socioeconómica, en función de una metodología basada en una regresión que aproxima la situación de pobreza y gastos/ingresos, la cual permite definir la inclusión/ exclusión de una persona en el SIS ${ }^{(19)}$. No obstante, han existido problemas relacionados a la implementación de los sistemas de información, de la validación con el Registro Nacional de Identidad, y la desarticulación entre el SIS y el SISFOH; lo cual podría verse reflejado en una disminución relativa de los afiliados el $2012{ }^{(15)}$. Asimismo, si bien en el Decreto Legislativo 1346 promulgado a inicios del 2017 se dictamina que todo afiliado al régimen subsidiado por afiliación regular deberá contar adicionalmente con su clasificación socioeconómica o ser considerado vulnerable, esta última condición, aunado al vencimiento de la clasificación socioeconómica, adolecen de falta de definición ${ }^{(20)}$.

Por su parte, en EsSalud advertimos un comportamiento distinto al SIS, con un incremento de la cobertura en la primera etapa (2009-2013) seguida de una fase de meseta (2013-2017). Esto se condice con la ampliación del trabajo asalariado formal en la década previa, principalmente en las zonas urbanas ${ }^{(21)}$, a la par de una reforma laboral que impulsó la creación de micro y pequeñas empresas ${ }^{(22)}$. Posterior a ello, notamos un estancamiento de la cobertura, lo cual podría explicarse por la desaceleración de la creación de nuevos empleos formales, con el crecimiento de relaciones laborales informales en algunas ramas de la actividad económica, que conllevarían a que grupos de trabajadores no accedan al régimen contributivo ${ }^{(23-24)}$. Es importante manifestar que la legislación peruana podría constituir una traba para la formalización del empleo, al existir regímenes laborales con índices de costo laboral por encima del $50 \%$, lo que podría conllevar a que empresas formales contraten personas bajo modalidades que impliquen una disminución de dichos costos, dentro de los cuales estaría el no pago de la seguridad social ${ }^{(22)}$. 
Adicionalmente, las barreras de acceso a la atención en la red de prestaciones de EsSalud, han sido precisadas como frenos para nuevas afiliaciones ${ }^{(25)}$

Lo encontrado es relevante, puesto que, si bien puede haber disimilitud entre la cobertura poblacional y la cobertura prestacional en el marco de la CUS ${ }^{(7)}$, existe evidencia primaria que reporta efectos positivos significativos del aseguramiento en materia de acceso a atención sanitaria en la población peruana ${ }^{(26)}$. Sin embargo, algunos autores han propuesto que la expansión de la protección financiera alcanzó un punto de saturación de los SESA, lo cual conllevó a la incapacidad de atender la totalidad de la demanda y a que una gran proporción de la población no utilice los servicios formales de salud en Perú ${ }^{25,27)}$.

Otro elemento para destacar es la distribución espacial de la cobertura, que en nuestro estudio muestra dos zonas diferenciadas. Por un lado, una franja que incluye a Lima, Ica y Arequipa, donde se concentra la mayor cantidad de proporción de población asegurada adscrita a EsSalud, en espacios urbanos, con una oferta orientada a servicios de alta complejidad y limitados centros del primer nivel de atención. Por otro lado, en el resto del país se encuentran altas coberturas por parte del SIS, con una gran cantidad de centros y puestos de salud, que cubren una alta proporción del territorio, pero con escaza capacidad resolutiva. Esta contradicción afecta significativamente la capacidad de operación tanto del MINSA-Gobiernos Regionales, como de EsSalud, y configura un escenario de desigualdad estructural ${ }^{(11)}$. No obstante, la reducción de las desigualdades va más allá de la articulación del aparato prestador público, e implica una visión integral del conjunto de factores, los cuales exceden el modelo de financiamiento, tales como las brechas de recursos humanos, la gobernanza, entre otros ${ }^{(12)}$.

Una arista importante corresponde a la perspectiva desde los DES. En ese contexto, la Superintendencia Nacional de Salud (SUSALUD), es el organismo técnico especializado adscrito al MINSA, que tiene como encargo del Estado peruano el desarrollar acciones en defensa de los DES de la población ${ }^{(28)}$. Si bien SUSALUD ha desplegado distintas estrategias en tema de aseguramiento, tales como el Registro Nominal de Afiliados y herramientas informáticas para facilitar la acreditación de los afiliados (29). Consideramos que SUSALUD en calidad de organismo supervisor y fiscalizador, podría evaluar si lo presentado en nuestro artículo muestran avances efectivos en el ejercicio de los DES; y en virtud de ello, podría desarrollar acciones de acuerdo con sus competencias. Al respecto, la Organización para la Cooperación y el Desarrollo Económicos (OCDE), señala que SUSALUD tiene un enorme potencial - y a la vez desafío - para constituirse como un motor de dinamización del sector, con incidencia en diferentes procesos que la articulan al SSP ${ }^{(12)}$.

Nuestro estudio presenta algunas limitaciones. Primero, dada la naturaleza transversal de los datos, no es posible evaluar un efecto causal; sin embargo, el conjunto de encuestas anuales conforma una serie de tiempoque permite valorar asociaciones con validez y confiabilidad. Segundo, los datos son obtenidos por autorreporte, por lo que podrían existir problemas de error de medición y sesgo de memoria; no obstante, la ENAHO es ejecutada por el INEI y cuenta con procedimientos estandarizados de control de calidad. Tercero, la eliminación de las personas que reportaron tener algún otro tipo de seguro de manera exclusiva podría generar un sesgo de selección; aunque, debemos precisar que solo fueron excluidos para los modelos multinomiales donde el reducido número de observaciones producía efectos negativos sobre la estabilidad de las regresiones múltiples. Cuarto, se midió el estar afiliado al SIS y EsSalud sin especificar el régimen correspondiente; sin embargo, la mayor proporción de afiliados son de naturaleza subsidiada y contributiva, respectivamente. Quinto, al tratarse de un análisis secundario de datos algunas variables importantes no fueron medidas por lo que no fueron incluidas en el análisis o construir otras variables de interés como es el caso de la pobreza multidimensional.

Finalmente, nuestro estudio es uno de los primeros que evalúa la evolución de la CAS entre los años 2009 al 2017, con énfasis en el SIS y EsSalud, dos de las principales IAFAS públicas del SSP. Consideramos perfiles diferenciados en los factores demográficos, sociales y económicos asociados a la condición de aseguramiento. Encontramos que nuestros resultados componen evidencia primaria de calidad que aporta elementos substanciales sobre el aseguramiento en salud para la toma de decisiones en Perú. Sugerimos investigaciones posteriores que analicen los efectos causales a distintos niveles y evalúen las relaciones con otros factores cardinales de la CUS en un marco de los DES.

Contribuciones de autores: EMH, LBB y JVO participaron en la concepción del estudio. EMH y LBB y JVO participaron del diseño de la ENSUSALUD (estudio primario). EMH, LBB, EA, JPM y JDC diseñaron el estudio. EMH, EA y BM realizaron el análisis de datos. Todos los autores interpretaron la información. EMH, EA, LBB y BM redactaron el borrador del manuscrito. JPM, JVO y JDC hicieron una revisión crítica y aportes substanciales al manuscrito. Todos los autores aprobaron la versión final y se responsabilizan del contenido del manuscrito.

Fuentes de financiamiento: este trabajo ha sido autofinanciado por los autores.

Conflicto de interés: LBB y JPM laboran en la Superintendencia Nacional de Salud (SUSALUD). JVO Y JDC han sido funcionarios en SUSALUD y en otras instituciones públicas relacionadas con el Aseguramiento en Salud. EMH ha laborado y ha realizado consultorías para SUSALUD y el Seguro Social en Salud (ESSALUD). EA y BM declaran no tener conflictos de interés.

Agradecimientos: los autores agradecen al Prof. Jay S. Kaufman (McGill University, Canadá) por su apoyo en la redacción del resumen en inglés.

Material suplementario: Disponible en la versión electrónica de la RPMESP. 


\section{REFERENCIAS BIBLIOGRÁFICAS}

1. Sen A. Why and how is health a human right? The Lancet.2008;372(9655):2010.

2. Ooms G, Latif LA, Waris A, Brolan CE, Hammonds R, Friedman EA, et al. Is universal health coverage the practical expression of the right to health care?. BMC Int Health Hum Rights. 2014;14:3.

3. Chapman AR. Assessing the universal health coverage target in the Sustainable Development Goals from a human rights perspective. BMC Int Health Hum Rights. 2016;16(1):33.

4. Atun R, de Andrade LOM, Almeida G, Cotlear D, Dmytraczenko T, Frenz P, et al. Health-system reform and universal health coverage in Latin America. The Lancet. 2015;385(9974):1230-47.

5. World Health Organization. Tracking universal health coverage: 2017 global monitoring report [Internet]. 2017. [citado el 10 de setiembre de 2018]. Disponible en: https://apps. who.int/iris/bitstream/handle/106 65/259817/9789241513555-eng. pdf; jsessionid $=5$ A741945EE198EB 951980C27DDF621A6? sequence $=1$.

6. Kieny MP, Bekedam H, Dovlo D, Fitzgerald J, Habicht J, Harrison G, et al. Strengthening health systems for universal health coverage and sustainable development. Bull World Health Organ. 2017;95(7):537-9.

7. Gutiérrez C, Romaní Romaní F, Wong P, Del Carmen Sara J. Brecha entre cobertura poblacional y prestacional en salud: un reto para la reforma de salud en el Perú. An Fac Med. 2018;79(1):65-70.

8. Frenk J, de Ferranti D. Universal health coverage: good health, good economics. Lancet Lond Engl. 2012;380(9845):862-4.

9. Salama P. Sistemas de protección social: entre la volatilidad económica y la vulnerabilidad social. Univ. Nacional de Colombia; 2005. 288 p.

10. Alcalde-Rabanal JE, Lazo-González O, Nigenda G. Sistema de salud de Perú. Salud Pública México. 2011;53:s243-54.

11. Sánchez-Moreno F. El sistema nacional de salud en el Perú. Rev Peru Med Exp Salud Publica. 2014;31(4):747-53.

12. Organisation for Economic Co-operation and Development. OECD Reviews of Health Systems: Peru 2017 [Internet]. OECD Publishing; 2017. [citado el 02 de setiembre de 2018]. Disponible en: http://www. oecd.org/countries/peru/oecd-reviews-of-health-systems-peru-20179789264282735-en.htm.

13. Wilson L, Velásquez A, Ponce C. La Ley Marco de Aseguramiento Universal en Salud en el Perú: análisis de beneficios y sistematización del proceso desde su concepción hasta su promulgación. Rev Peru Med Exp Salud Pública. 2009;26(2):207-217.

14. Velásquez A, Suarez D, Nepo-Linares E. Reforma del sector salud en el Perú: derecho, gobernanza, cobertura universal y respuesta contra riesgos sanitarios. Rev Peru Med Exp Salud Publica. 2016;33(3):546-55.

15. Petrera Pavone M. El complejo proceso de la inclusión: reforma y seguro integral de salud. Lima: Universidad Peruana Cayetano Heredia, Fondo Editorial; 2018. 141 p.

16. Education attainment - Population with tertiary education - OECD Data [Internet]. [Citado el 12 de octubre de 2018]. Disponible en: https://data. oecd.org/eduatt/population-with-tertiary-education.htm.

17. Hosmer DW, Lemeshow S. Chapter 8: Special Topics. The Multinomial logistic regression model. In: Applied Logistic Regression. John Wiley \& Sons; 2004.

18. Ministerio de Desarrollo e Inclusión Social. Una política para el desarrollo y la inclusión social en el Perú [Internet]. 2012. [citado el 16 de setiembre de 2018]. Disponible en: http://www. midis.gob.pe/files/doc/midis_politicas_desarrollo_es.pdf.

19. Ministerio de Desarrollo e Inclusión Social. Metodología para la determinación de la clasificación socioeconómica [Internet]. Lima: MIDIS; 2016 [citado el 10 de octubre de 2018]. Disponible en: http://www.midis.gob.pe/ index.php/es/principales-contenidos/ doc_download/5177-resolucion-ministerial-n-151-2016-midis.

20. Perú. Ministerio de Salud. Decreto Legislativo $\mathrm{N}^{\circ} 1346$. Decreto Legislativo que establece disposiciones para optimizar los servicios que son financiados a través del Seguro Integral de Salud.

21. Cetrángolo O, Bertranou F, Casanova L, Casalí P, International Labour Office, EsSalud (Peru). El sistema de salud del Perú: situación actual y estrategias para orientar la extensión de la cobertura contributiva. Lima: OIT: EsSalud; 2013.
22. Chacaltana J. Perú, 2002-2012: crecimiento, cambio estructural y formalización [Internet]. Lima; 2016 [citado el 22 de octubre de 2018]. Disponible en: https://repositorio.cepal.org/handle/11362/40402.

23. Chacaltana J, Ruiz C. El mercado laboral peruano y el futuro del trabajo. Relac Laborales Derecho Empl. 2017;5(1):1-35.

24. Enciso A, Manuel A. Empleo informal y mercados laborales multisegmentados en el Perú. Pontif Univ Católica Perú [Internet]. 2018 [Citado el 22 de octubre de 2018]. Disponible en: http:// tesis.pucp.edu.pe/repositorio//handle/123456789/12366.

25. Benites-Zapata VA, Lozada-Urbano M, Urrunaga-Pastor D, Márquez-Bobadilla E, Moncada-Mapelli E, Mezones-Holguin E. Factores asociados a la no utilización de los servicios formales de prestación en salud en la población peruana: análisis de la Encuesta Nacional de Hogares (ENAHO) 2015. Rev Peru Med Exp Salud Pública. 2017;34(3):478-84.

26. Neelsen S, O’Donnell O. Progressive universalism? The impact of targeted coverage on health care access and expenditures in Peru. Health Econ. 2017;26(12):e179-203.

27. Durán V. Estudio financiero-actuarial y de la gestión de EsSalud. Análisis y recomendaciones técnicas. Organización Internacional del trabajo; 2005.

28. Mezones-Holguín E, Díaz-Romero R, Castillo-Jayme J, Jerí-de-Pinho M, BenitesZapata V, Marquez-Bobadilla E, et al. Promoción de los derechos en salud en Perú: una aproximación desde la perspectiva de acción de la Superintendencia Nacional de Salud. Rev Peru Med Exp Salud Pública. 2016;33(3):520-8.

29. Villegas-Ortega J, Loyola-Martínez C, Santisteban-Romero J, ManchegoLombardi M, Lozada-Urbano M. Tecnologías de la información para resolver contingencias en la afiliación al régimen subsidiado de salud en Perú: "Resuelve tu afiliación". Rev Peru Med Exp Salud Publica. 2016;33(3):561-6.

Correspondencia: Edward Mezones Holguin. Dirección: Avenida la Fontana 550, La Molina, Lima, Perú.

Correoeléctronico:emezones@usil.edu.pe emezones@gmail.com 